Marquette University

e-Publications@Marquette

Biological Sciences Faculty Research and

Publications

Biological Sciences, Department of

$10-1-2010$

\title{
Circadian Disruption and Metabolic Disease: Findings from Animal Models
}

Deanna M. Arble

Marquette University, deanna.arble@marquette.edu

Kathryn Moynihan Ramsey

Northwestern University

Joseph Bass

Norhtwestern University

Fred W. Turek

Northwestern University

Accepted version. Best Practice and Research Clinical Endocrinology and Metabolism, Vol. 24, No. 5 (October 2010): 785-800. DOI. (C) 2010 Elsevier B.V. Used with permission.

Deanna Marie Arble was affiliated with Northwestern University at the time of publication. 
Marquette University

\section{e-Publications@Marquette}

\section{Biology Faculty Research and Publications/College of Arts and Sciences}

This paper is NOT THE PUBLISHED VERSION; but the author's final, peer-reviewed manuscript. The published version may be accessed by following the link in the citation below.

Surgery for Obesity and Related Diseases, (2018). DOI. This article is (C) Elsevier and permission has been granted for this version to appear in e-Publications@Marquette. Elsevier does not grant permission for this article to be further copied/distributed or hosted elsewhere without the express permission from Elsevier.

\section{Contents}

Highlights

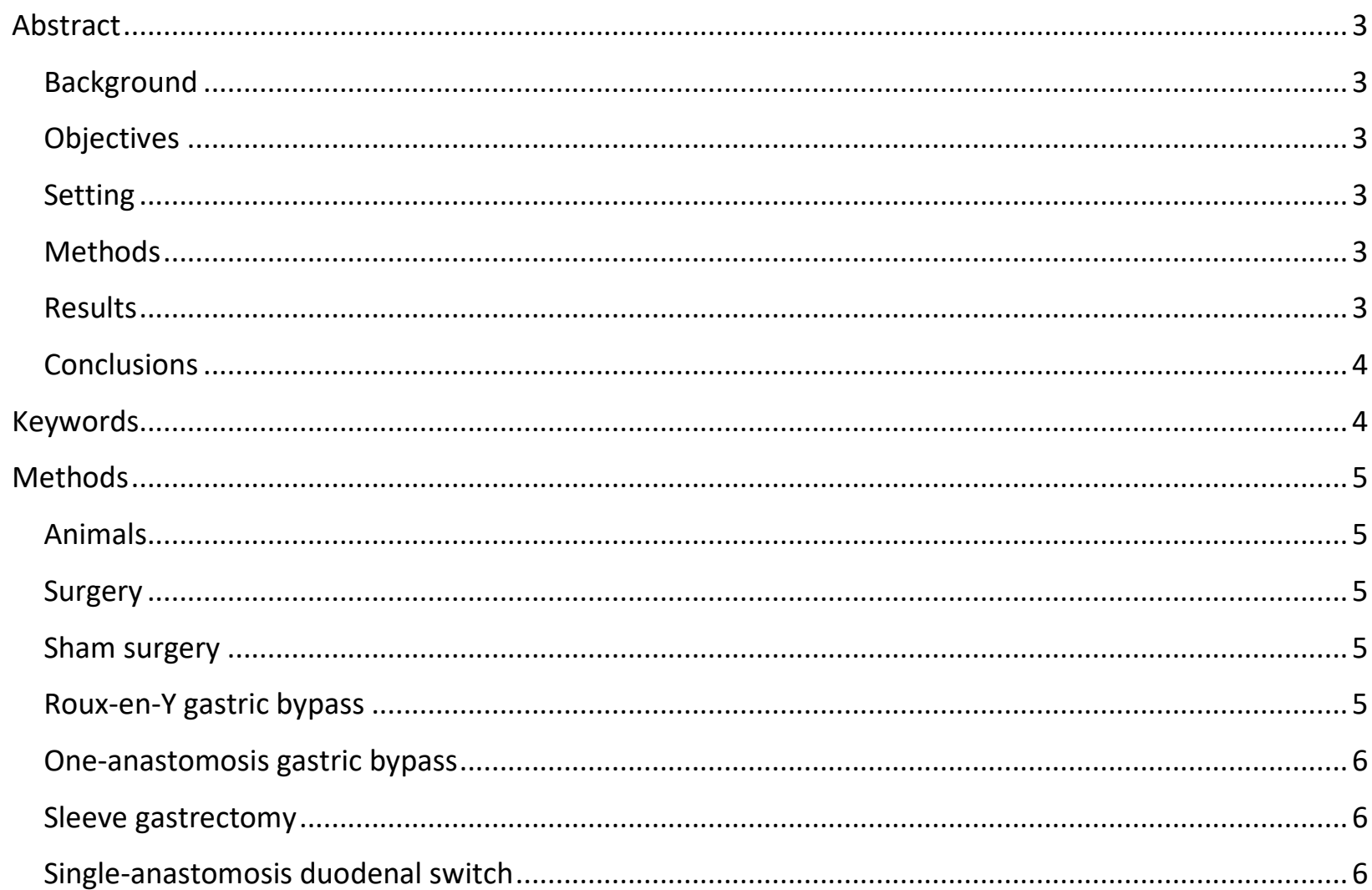




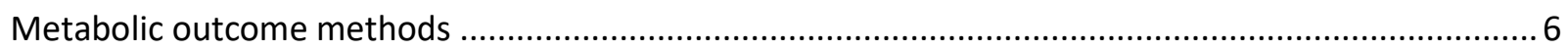

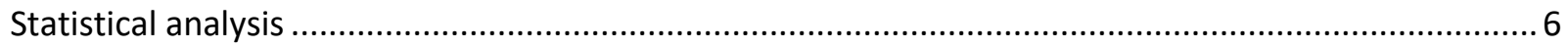

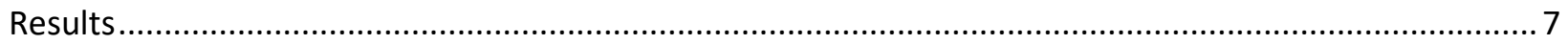

Surgical effect on body mass and body composition ..................................................................... 7

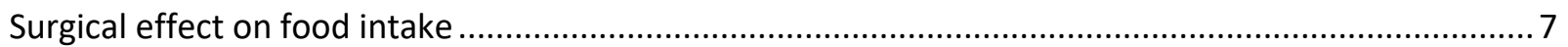

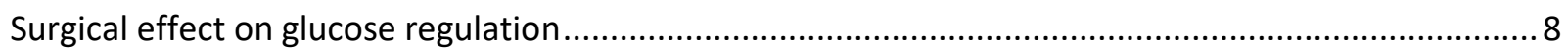

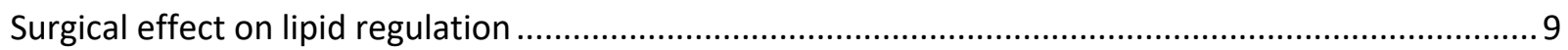

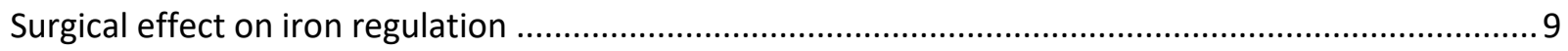

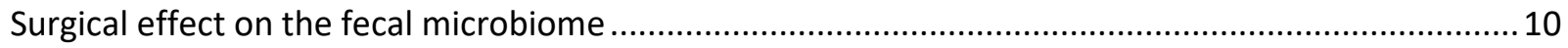

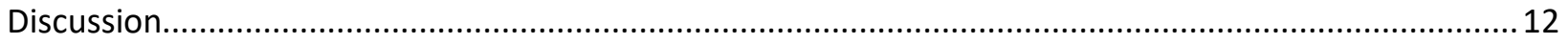

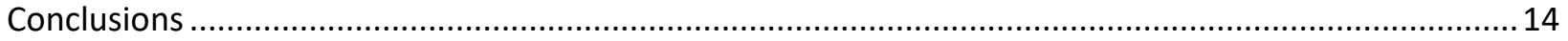

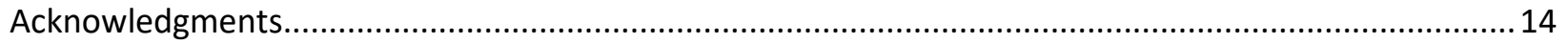

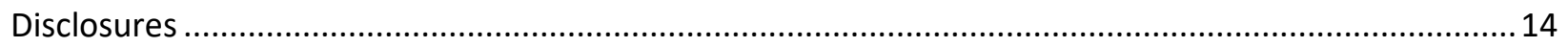

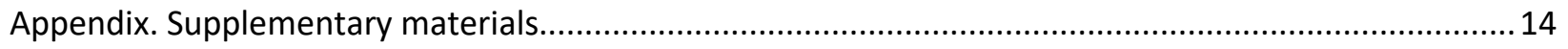

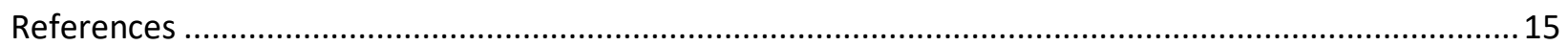

\section{Metabolic comparison of one-anastomosis gastric bypass, single-anastomosis duodenal-switch, Roux-en-Y gastric bypass, and vertical sleeve gastrectomy in rat}

\section{Deanna M. Arble}

Department of Surgery, University of Michigan, Ann Arbor, MI

Department of Biological Sciences, Marquette University, Milwaukee, WI

Simon S. Evers

Department of Surgery, University of Michigan, Ann Arbor, MI

Nadejda Bozadijeva

Department of Surgery, University of Michigan, Ann Arbor, MI

Henriette Frikke-Schmidt

Department of Surgery, University of Michigan, Ann Arbor, MI 


\section{Andriy Myronovych}

Department of Surgery, University of Michigan, Ann Arbor, MI

Alfor Lewis

Department of Surgery, University of Michigan, Ann Arbor, MI

Mouhamadoul Habib Toure

Department of Surgery, University of Michigan, Ann Arbor, MI

Randy J. Seeley

Department of Surgery, University of Michigan, Ann Arbor, MI

\section{Highlights}

- $\quad$ Diet induced obese rat models of OAGB, SADS, RYGB, and SG are suitable preclinical models. Weight loss and improved glucose dynamics are similar to those in the clinic.

- OAGB results in metabolic improvements that met or exceed RYGB.

- Each surgical procedure results in a specific metabolic signature - including differential changes in food intake, insulin, GLP-1 response, iron regulation, and fecal microbiota.

\section{Abstract}

\section{Background}

One-anastomosis gastric bypass (OAGB) and single-anastomosis duodenal switch (SADS) have become increasingly popular weight loss strategies. However, data directly comparing the effectiveness of these procedures with Roux-en-Y gastric bypass (RYGB) and vertical sleeve gastrectomy (SG) are limited.

\section{Objectives}

To examine the metabolic outcomes of OAGB, SADS, RYGB, and SG in a controlled rodent model.

\section{Setting}

Academic research laboratory, United States.

\section{Methods}

Surgeries were performed in diet-induced obese Long-Evans rats, and metabolic outcomes were monitored before and for 15 weeks after surgery.

\section{Results}

All bariatric procedures induced weight loss compared with sham that lasted throughout the course of the study. The highest percent fat loss occurred after OAGB and RYGB. All bariatric procedures had improved glucose dynamics associated with an increase in insulin (notably OAGB and SADS) and/or glucagon-like protein-1 secretion. Circulating cholesterol was reduced in OAGB, SG, and RYGB. OAGB and SG additionally decreased circulating triglycerides. Liver triglycerides were most profoundly reduced after OAGB and RYGB. Circulating iron levels were decreased in all surgical groups, associated with a decreased hematocrit value and increased reticulocyte count. The fecal microbiome communities of 
OAGB, SADS, and RYGB were significantly altered; however, SG exhibited no change in microbiome diversity or composition.

\section{Conclusions}

These data support the use of the rat for modeling bariatric surgical procedures and highlight the ability of the OAGB to meet or exceed the metabolic improvements of RYGB. These data point to the likelihood that each surgery accomplishes metabolic improvements through both overlapping and distinct mechanisms and warrants further research.

\section{Keywords}

One-anastomosis gastric bypass; Single-anastomosis duodenal switch; Roux-en-Y gastric bypass; Sleeve gastrectomy; Rodent; Animal model; Glucose regulation

Roux-en-Y gastric bypass (RYGB) and sleeve gastrectomy (SG) are the most commonly performed bariatric surgical procedures $\underline{1}$ and result in significant and sustained weight loss accompanied by dramatic changes in glucose metabolism. ${ }^{2,3}$ However, surgeons continue to explore other procedures that may carry advantages in terms of surgical approach or metabolic improvements. Among these procedures are the one-anastomosis gastric bypass (OAGB), also known as the mini bypass or the omega loop gastric bypass, and the single-anastomosis duodenal switch (SADS), both of which produce significant improvements in weight and glucose homeostasis. $\frac{., \underline{5}}{}$

Surgeons drawing observations from patients have dominated the development of these bariatric procedures. Nonetheless, the utilization of animal models is an important step in pinpointing the surgical effects on metabolism independent of genetic background, diet adherence, and environmental conditions. By allowing investigators to test a wide range of hypotheses while limiting variability compared with the human population, animal models enable the identification of mechanistic hypotheses to explain the potent metabolic effects of bariatric surgery. Of note, the use of animal models in this study confers the following 2 important benefits over clinical studies: (1) when possible, we standardize gut manipulations to draw meaningful comparisons on how specific changes to gut physiology impact metabolism; and (2) as animal models do not follow clinician advice on diet and/or exercise, any observed outcomes are due entirely to the surgical procedure and its effect on the brain and overall physiology. An important validity measure of these animal models depends on the similarity in postsurgical responses compared with the patient population at the level of weight maintenance and metabolic outcome.

Indeed, the mechanisms by which bariatric surgical procedures result in weight loss and metabolic improvements are still not completely understood. A number of hypotheses have been forwarded to explain these metabolic improvements, including the involvement of glucose-regulating gut hormones, such as glucagon-like protein-1 (GLP-1),,$-\underline{6}$ bile acids, $, 8,9$ and alterations in the microbiome. ${ }^{10,11}$ By comparing different bariatric surgical procedures in obese rats, we can systemically investigate the physiologic components correlated with metabolic success and how they relate to specific physical changes of the surgery. Moreover, we can identify what surgical intervention may be more effective for a specific metabolic outcome in a controlled, nonvariable setting. Here, we present evidence that our rodent procedure similar to OAGB meets or exceeds the metabolic improvements of the comparative 
gold standard bariatric surgical procedure, the RYGB. Moreover, each bariatric surgical procedure leads to differential effects on metabolism, and when viewed in light of the surgical approach, suggests that a combination of overlapping and distinct mechanisms may contribute to the general success of bariatric surgery.

\section{Methods}

\section{Animals}

Male Long-Evans rats (Envigo, Indianapolis, IN, USA) were maintained on a high-fat diet with butter fat (40\% fat, $4.54 \mathrm{kcal} / \mathrm{g}$; D03082706 Research Diets, New Brunswick, NJ, USA) to induce obesity before bariatric surgery. Before surgery, all rats received baseline measurement of weight and body composition. After surgery, rats were maintained on the same high-fat diet. Weight, food intake, and body composition were measured periodically over 15 weeks. The number of animals per group that survived until the end of the study were as follows: sham (7/9), RYGB (7/13), OAGB (6/13), SG (12/13), and SADS (9/13). Rats were excluded from analysis for failure to recover from the surgery or for exhibiting signs of intestinal obstruction or infection. Rats were individually housed in temperaturecontrolled rooms with a 12:12-hour light cycle. All studies were approved by and performed according to the guidelines of the Institutional Animal Care and Use Committee of the University of Michigan.

\section{Surgery}

After induction of isoflurane anesthesia, animals received buprenorphine hydrochloride $(.03 \mathrm{mg} / \mathrm{kg})$, meloxicam $(.5 \mathrm{mg} / \mathrm{kg})$, gentamicin $(8 \mathrm{mg} / \mathrm{kg})$, and $10 \mathrm{~mL}$ isotonic saline, all by subcutaneous injection. All surgeries began with a midline abdominal skin incision, followed by an incision in the underlying muscle wall. All anastomoses were performed with 8-0 Prolene sutures (Ethicon, Somerville, NJ, USA) and abdominal closures were performed in 2 layers with 4-0 Vicryl Rapide sutures (Ethicon). Postoperatively, animals received a liquid diet (Osmolite1 Cal, Abbott Nutrition, Abbott Laboratories; Columbus, $\mathrm{OH}$, USA) for 4 days before being returned to the previously described high-fat diet. All animals received subcutaneous injection of buprenorphine hydrochloride twice daily and meloxicam and gentamicin once daily for 3 days.

\section{Sham surgery}

The stomach was exposed, gentle pressure applied with forceps, and the stomach was covered with a gauze pad soaked in sterile saline. The stomach was returned to the body cavity and the incision closed.

\section{Roux-en-Y gastric bypass}

The stomach and the intestines were exposed and draped on a gauze pad soaked in sterile saline. A triangular stomach pouch of reduced volume was created via 2 intersecting cuts using an endoscopic stapler (Endopath ETS-Flex 35 mm Endoscopic Articulating Linear Cutter; Ethicon Endo-Surgery Inc., Cincinnati, $\mathrm{OH}, \mathrm{USA}$ ) to separate the pouch from the remnant stomach. The jejunum was transected 30 $\mathrm{cm}$ distal to the ligament of Treitz, and a gastrotomy incision was made in the stomach pouch. The transected end of the distal portion of the jejunum was anastomosed to the gastrotomy (forming the Roux $\operatorname{limb}$ ) with interrupted sutures. The remaining transected end of the proximal portion of the intestine was then anastomosed with interrupted sutures to the Roux limb as an end-to-side jejunojejunostomy at $10 \mathrm{~cm}$ from the gastrojejunostomy. 


\section{One-anastomosis gastric bypass}

The stomach and the intestines were exposed and draped on a gauze pad soaked in sterile saline. A stomach pouch of reduced volume was created as described for RYGB. The jejunum at $30 \mathrm{~cm}$ from the ligament of Treitz was brought up to contact the stomach pouch, and incisions of comparable length were made in both. The jejunum was anastomosed to the stomach pouch with interrupted sutures. Note, we have selected a limb length similar to our RYGB procedure to make meaningful comparisons about the effect of a single anastomosis on metabolic outcomes. Thus, this method deviates from clinical OAGB in that OAGB procedures traditionally have a longer limb length than that of RYGB procedures.

\section{Sleeve gastrectomy}

The stomach was exposed and transected to form a sleeve using an endoscopic stapler (ETS-Flex 35; Ethicon). Approximately $70 \%$ of the rat stomach (by weight) was removed, including the majority of the fundus and about $50 \%$ of the pyloric antrum.

\section{Single-anastomosis duodenal switch}

The stomach was exposed and covered with a gauze pad soaked in sterile saline. The duodenum was identified and transected proximally to where the common bile duct enters; the free end of the distal duodenum was sutured. The gauze was removed from the stomach, which was transected to form a sleeve as described for SG. The jejunum (at $30 \mathrm{~cm}$ from the ligament of Treitz) was mobilized to contact the proximal duodenal free end and an incision was made in the antimesenteric side of the former to match its length to the duodenal diameter. The jejunum was connected to the proximal duodenal segment by side-to-end anastomosis with interrupted sutures. As with the OAGB procedure, we have selected a limb length similar to our RYGB procedure to make meaningful comparisons about the effect of intestinal rerouting in the context of a reduced stomach size on metabolic outcomes. Thus, this method deviates from clinical SADS in that SADS procedures traditionally have a much longer limb length than that of RYGB procedures.

\section{Metabolic outcome methods}

Detailed methods on metabolic outcome measures, including body composition, mixed-meal glucose tolerance tests, total GLP-1 measures, lipid analysis, iron regulation, and fecal microbiome can be found in supplementary methods.

\section{Statistical analysis}

Statistical analysis was performed using GraphPad Prism (GraphPad, La Jolla, USA). Unless otherwise specified, we used one-way analysis of variance and Tukey post hoc tests where necessary to determine significant differences between groups. We used a repeated-measures analysis of variance for analysis of weight and food intake over time with a Bonferroni's multiple comparison test when a significant interaction effect was found. All statistical analysis used a 2-tailed design and results were considered significant when $P<.05$. 


\section{Results}

\section{Surgical effect on body mass and body composition}

In comparison to the control sham surgery, all bariatric surgical groups lost weight and maintained a lower weight for the duration of the 15-week study (106 d). The RYGB and OAGB groups lost the most weight, whereas SG and SADS groups lost an intermediate amount of weight (Figs. 1A, 1B). Post hoc analysis on total weight loss revealed that all groups lost more weight than sham $(P<.001)$, and RYGB lost significantly more weight than SG $(P<.05)$.

A

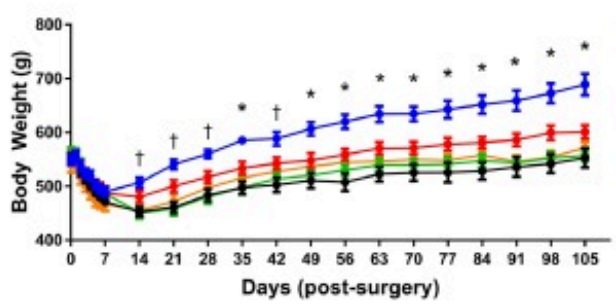

C

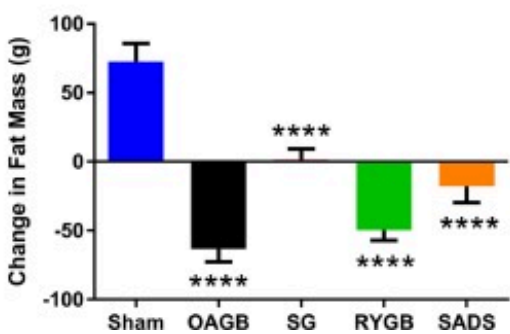

B
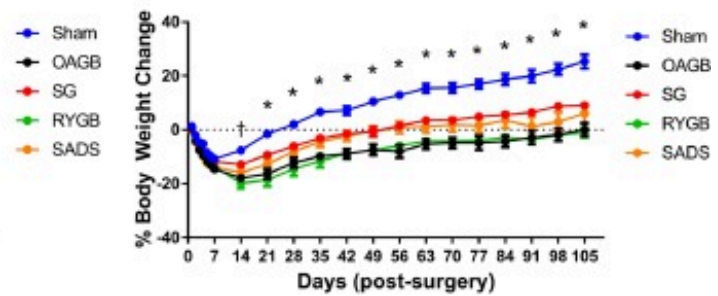

D

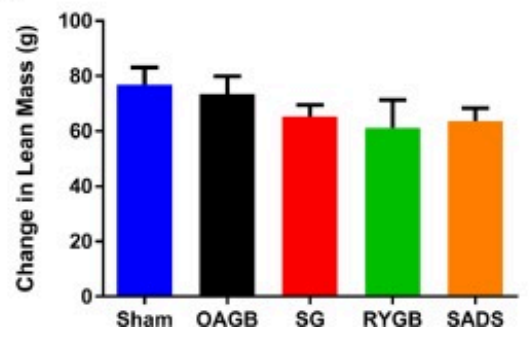

Fig. 1. Surgical effect on body mass and body composition. (A) Absolute weight changes (in g) after surgery. *All groups differ from sham; tone-anastomosis gastric bypass, Roux-en-Y gastric bypass, and single-anastomosis duodenal switch differ from sham. (B) Percent change from baseline weight after surgery. *All groups differ from sham; †one-anastomosis gastric bypass, Roux-en-Y gastric bypass, and single-anastomosis duodenal switch differ from sham. (C) Absolute change in fat mass 15 weeks postsurgery. ${ }^{* * * *}$ Significant difference from Sham, $P$ $<.0001$. (D) Absolute change in lean mass 15 weeks postsurgery.

Body fat composition was similarly altered 15 weeks after surgery, with all groups losing a significant amount of fat mass compared with sham (Fig. 1C). The largest amount of fat loss occurred after OAGB and RYGB, with SADS and SG exhibiting an intermediate amount of fat loss (Fig. 1C). Post hoc analysis revealed that OAGB lost significantly more fat mass than SG $(P<.001)$ and SADS $(P<.05)$. RYGB lost significantly more fat mass than SG $(P<.01)$.

Surgery did not affect change in lean mass (Fig. 1D), and there were no significant differences in baseline fat or lean mass (Supplemental Fig. 1).

\section{Surgical effect on food intake}

Rats were maintained on a high-fat diet before and after surgery. Cumulative food intake, as measured from surgical recovery to 14 weeks ( $98 \mathrm{~d}$ ) postsurgery, was reduced after the OAGB and SG compared with sham (Fig. 2A). Cumulative food intake was not reduced in RYGB or SADS compared with shamoperated animals (Fig. 2A). All surgical groups exhibited a transient decrease in food intake 2 weeks (SG, $P<.001 ;$ OAGB, RYGB, SADS, $P<.0001$ ) and 3 weeks (SADS, $P<.01 ; \mathrm{SG}, P<.001 ;$ OAGB, RYGB, $P<.0001$ ) 
after surgery compared with sham-operated controls (Fig. 2B). Notably, the OAGB and SG groups, which also had significant reductions in cumulative intake, exhibited longer transient reductions in food intake of approximately 6 weeks ( $42 \mathrm{~d}$ ) in duration.

A

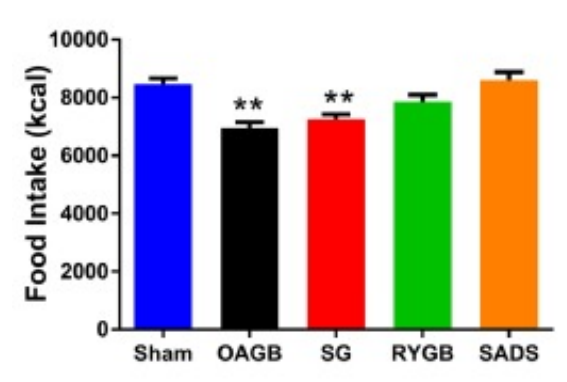

B

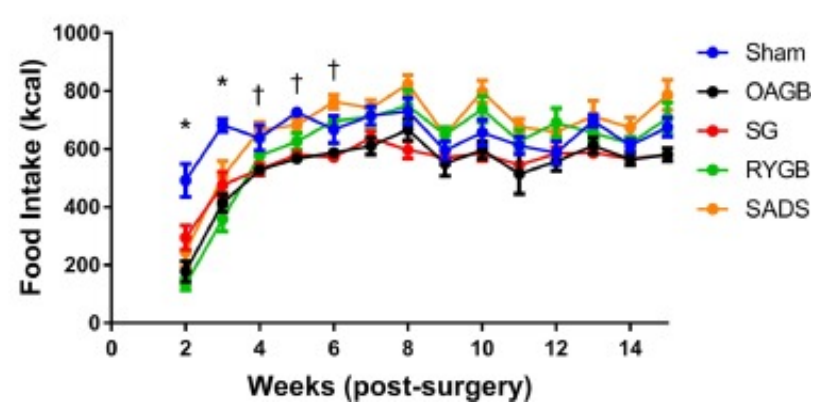

Fig. 2. Surgical effect on food intake. (A) Cumulative caloric intake from surgical recovery to 14 weeks postsurgery. **Significant difference from sham, $P<.01$ (B) Weekly caloric intakes of each surgical group; *All groups differ from sham; tone-anastomosis gastric bypass and sleeve gastrectomy differ from sham.

\section{Surgical effect on glucose regulation}

Fasting glucose levels were only decreased in the SG compared with sham controls (Fig. 3A). In response to an oral mixed-meal glucose tolerance test, all surgical groups had improved glucose clearance and lower glucose levels at 30,45, and 60 minutes after the oral load compared with sham-operated controls (Fig. $3 B$ ). The rise in blood glucose (e.g., the difference between the 15 - min time point and the baseline) was significantly greater in OAGB $(P<.01)$, RYGB $(P<.05)$, and SADS $(P<.05)$ compared with the glucose rise experienced by sham-operated controls (Fig. $3 B$ ).

A

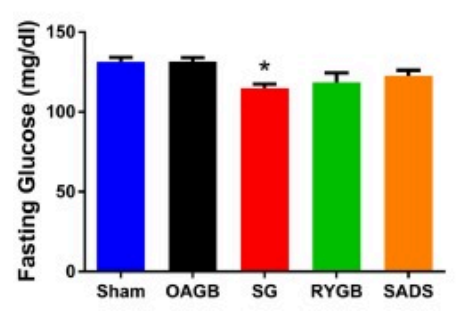

C

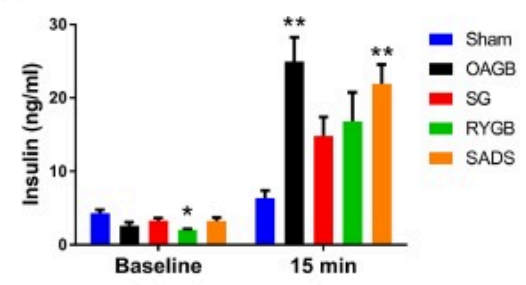

B

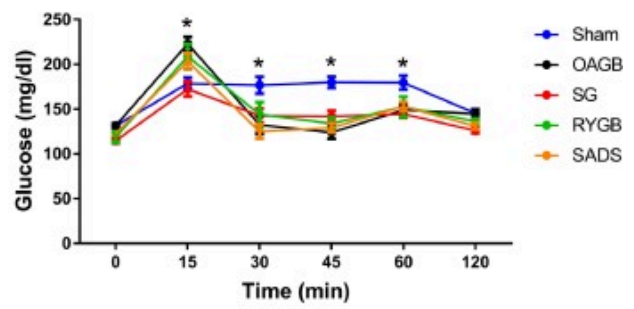

D

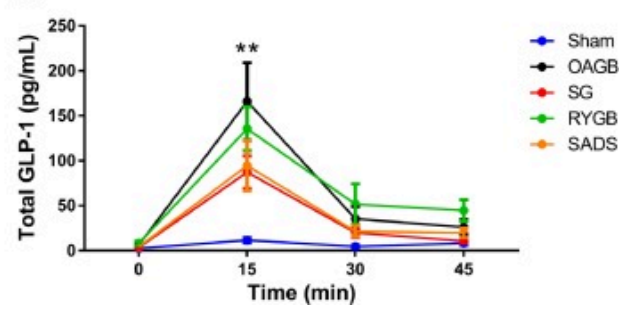

Fig. 3. Surgical effect on glucose regulation. (A) Fasting blood glucose levels. *Significant difference from sham, $P$ $<.05$. (B) Glucose response to an oral mixed-meal tolerance test. *Significant difference from sham, $P<.05$. (C) Baseline/fasting plasma insulin levels and plasma insulin 15 minutes after an oral mixed meal. *Significant difference from sham at same time point, $P<.05, * * P<.01, * * * * P<.0001$. (D) Total glucagon-like protein-1 response to an oral mixed-meal tolerance test. *All groups significant difference from sham, $P<.05$. 
Baseline insulin levels were only decreased in the RYGB compared with sham controls (Fig. $3 \mathrm{C})$. Although all surgical groups exhibited an increase in insulin 15 minutes after an oral mixed-meal load compared with baseline, only OAGB and SADS had a glucose-stimulated insulin response greater than sham (Fig. $\underline{3} C)$. Using a repeated-measures analysis of variance, total GLP-1 in plasma was significantly increased 15 minutes after an oral mixed-meal in all surgical groups compared with sham $(P<.01$; Fig. 3D). Moreover, post hoc analysis with Sidak's multiple comparison test revealed OAGB had increased total GLP-1 compared with SG $(P<.01)$ and SADS $(P<.01)$ at 15 minutes postgavage.

\section{Surgical effect on lipid regulation}

Circulating ad libitum plasma levels of cholesterol were significantly reduced in rats undergoing OAGB, SG, and RYGB compared with sham (Fig. 4A). No differences were observed in circulating levels of nonesterified fatty acids (Fig. 4B). Plasma triglyceride levels were reduced after OAGB and SG compared with sham (Fig. 4C). While there were no significant reductions in plasma triglycerides in the RYGB or SADS groups, there was a significant reduction in liver triglycerides (Figs. 4C, 4D). Indeed, liver triglycerides were significantly reduced in all bariatric surgical groups compared with sham (Fig. 4D). Moreover, post hoc analysis revealed that OAGB $(P<.01)$, RYGB $(P<.001)$, and SADS $(P<.05)$ had lower liver triglycerides compared with SG.
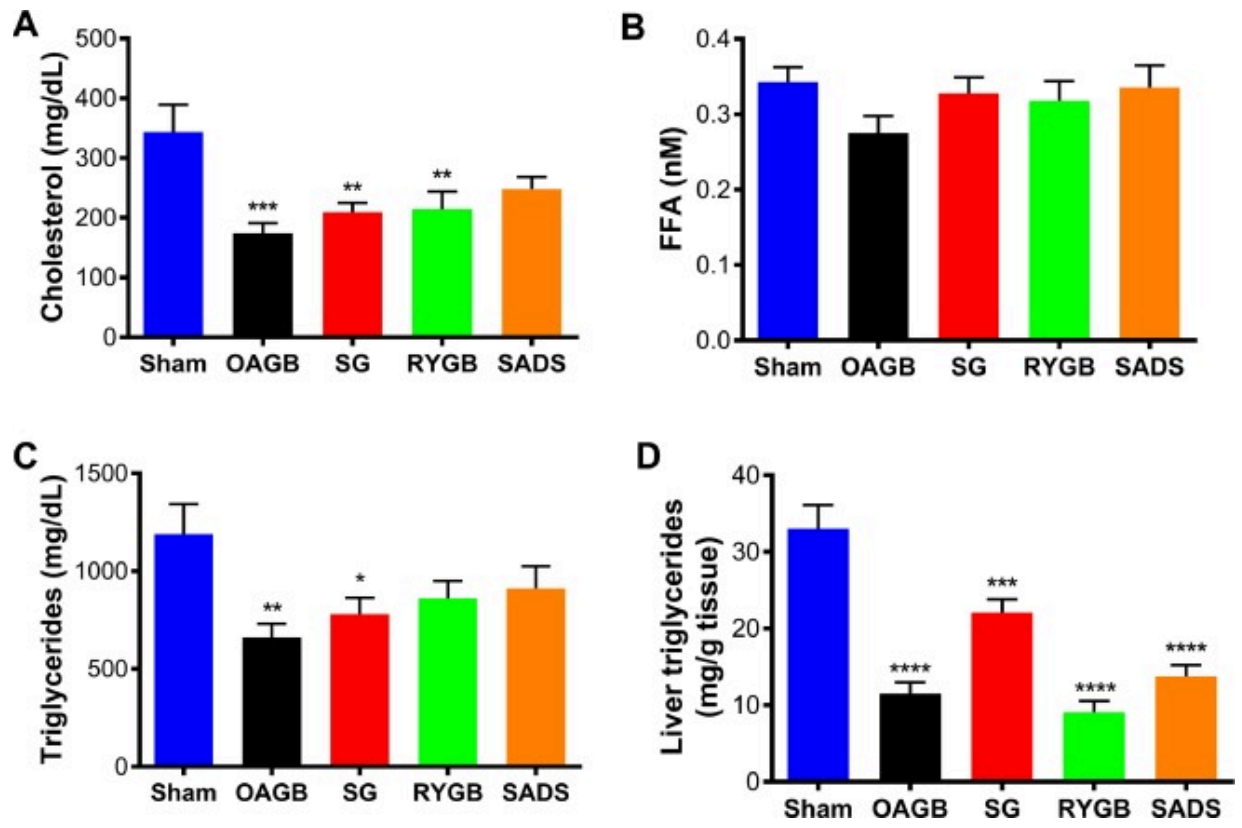

Fig. 4. Surgical effect on lipid regulation. (A) Plasma cholesterol levels. ${ }^{* *}$ Significant difference from sham, $P<.01$. (B) Plasma free fatty acids (FFA). (C) Plasma triglycerides. *Significant difference from sham, $P<.05, * * P<.01$. (D) Liver triglycerides expressed as milligram per gram of liver tissue. ${ }^{* * *}$ Significant difference from sham, $P<.001$, $* * * * P<.0001$.

\section{Surgical effect on iron regulation}

Compared with sham-operated controls, total circulating iron levels were reduced in all surgery groups except for RYGB (Fig. 5A). Hematocrit was reduced in rats undergoing SADS, RYGB, and OAGB compared with sham (Fig. 5B). Moreover, post hoc analysis revealed hematocrit of OAGB was significantly reduced compared with SG $(P>.001)$. Although a trend was observed for an increase in reticulocyte count in all 
groups, only SADS- and SG-operated rats had a significantly elevated reticulocyte count compared with sham (Fig. 5C).

A

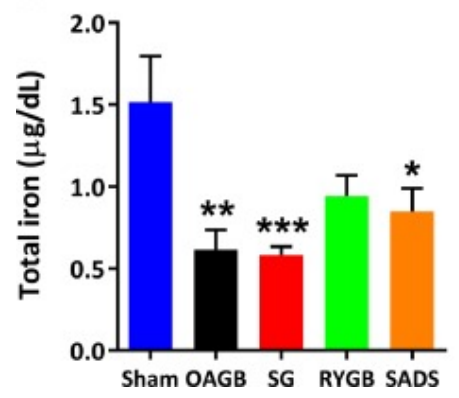

B

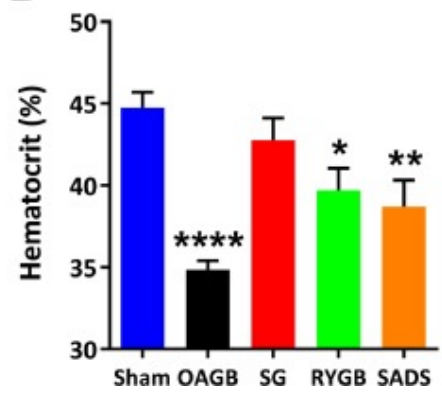

C

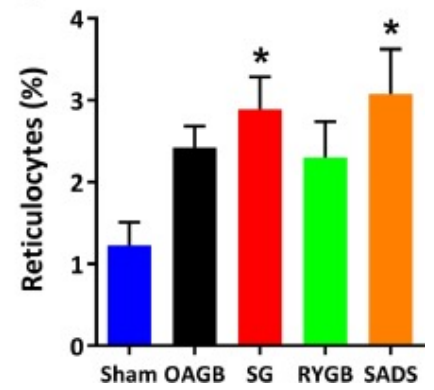

Fig. 5. Surgical effect on iron regulation. (A) Total circulating iron levels. *Significant difference from sham, $P<.05$, $* * P<.01, * * * P<.001$. (B) Hematocrit levels, *Significant difference from sham, $P<.05, * * P<.01, * * * * P<.0001$. (C) Reticulocyte count. *Significant difference from sham, $P<.05$.

\section{Surgical effect on the fecal microbiome}

$\theta_{\mathrm{yc}}$ was used to determine the ability of the surgery to procedure distinct microbiome communities. $\theta_{\mathrm{Yc}}$ distances were significantly large for all surgical comparisons with sham except SG versus sham (Wilcoxon test $P<.05$ ), pointing to distinct microbiome communities occurring within all surgeries except SG (Fig. 6A, Table 1). Lack of significance in the SG group may be related to the variability in postsurgical weight. Specifically, axis 2 of $\theta_{\mathrm{yc}}$ significantly predicted 15 -week postsurgical weight within the SG group $\left(R^{2}=.35, \mathrm{~F}_{1,10}=5.32, P<.05\right)$. In particular, 8 operational taxonomic units (OTUs) with an linear discriminant analysis (LDA) score $>4$ were identified as differently abundant across the surgical groups (Table 2). However, no significant differences were observed in microbiome diversity or richness

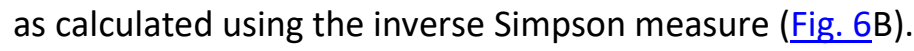

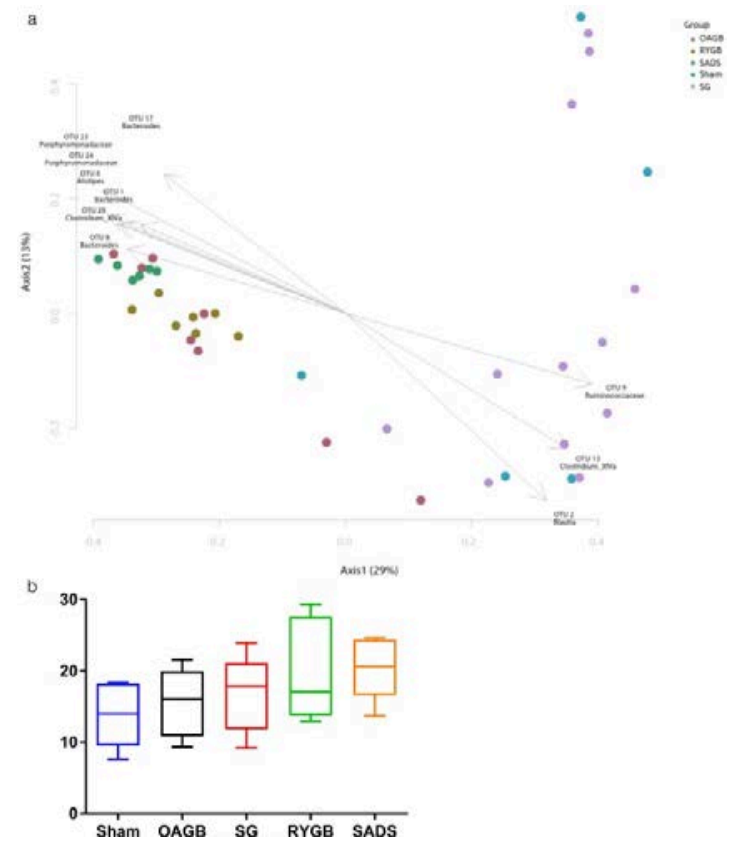

Fig. 6. Surgical effect on the fecal microbiome. (A) There were significant differences in the microbiota of most surgical groups, as calculated by measuring the distance between communities $\left(\theta_{\mathrm{rc}}\right)$. Only sleeve gastrectomy 
versus sham was found to be similar in microbiome population. (B) No significant differences were observed in microbiome diversity as calculated using the inverse Simpson measure.

Table 1. Surgical effect on the fecal microbiome population

\begin{tabular}{|l|l|l|l|l|}
\hline & OAGB & SG & RYGB & SADS \\
\hline Sham & $<.001$ & .216 & .002 & .001 \\
\hline OAGB & & $<.001$ & .046 & .029 \\
\hline SG & & & $<.001$ & $<.001$ \\
\hline RYGB & & & & .013 \\
\hline
\end{tabular}

$\mathrm{OAGB}=$ one-anastomosis gastric bypass; SADS = single-anastomosis duodenal switch; RYGB = Roux-en-Y gastric bypass; SG = sleeve gastrectomy (vertical).

$P$ value comparison of $\theta_{\mathrm{rc}}$ values across surgical groups.

Table 2. Surgical effect on the fecal microbiome population

\begin{tabular}{|c|c|c|c|}
\hline LDA & $P$ value & Group & Taxonomy \\
\hline 4.4893 & $1.01^{\mathrm{E}-05}$ & RYGB & $\begin{array}{l}\text { Bacteria; bacteroidetes; bacteroidia; bacteroidales; rikenellaceae; } \\
\text { alistipes }\end{array}$ \\
\hline 4.55226 & $2.46^{\mathrm{E}-06}$ & SG & Bacteria; firmicutes; clostridia; clostridiales; ruminococcaceae \\
\hline 4.35383 & $2.23^{\mathrm{E}-05}$ & Sham & $\begin{array}{l}\text { Bacteria; firmicutes; clostridia; clostridiales; lachnospiraceae; } \\
\text { clostridium_XIVa }\end{array}$ \\
\hline 4.33306 & $4.59^{\mathrm{E}-05}$ & SADS & $\begin{array}{l}\text { Bacteria; firmicutes; clostridia; clostridiales; lachnospiraceae; } \\
\text { clostridium_XIVa }\end{array}$ \\
\hline 4.19178 & $1.35^{\mathrm{E}-05}$ & SADS & $\begin{array}{l}\text { Bacteria; firmicutes; clostridia; clostridiales; lachnospiraceae; } \\
\text { clostridium_XIVa }\end{array}$ \\
\hline 4.27848 & $6.72^{\mathrm{E}-06}$ & SADS & $\begin{array}{l}\text { Bacteria; bacteroidetes; bacteroidia; bacteroidales; } \\
\text { porphyromonadaceae }\end{array}$ \\
\hline 4.05671 & $2.63^{\mathrm{E}-05}$ & OAGB & $\begin{array}{l}\text { Bacteria; bacteroidetes; bacteroidia; bacteroidales; } \\
\text { porphyromonadaceae; barnesiella }\end{array}$ \\
\hline 4.26218 & $1.70^{\mathrm{E}-06}$ & Sham & Bacteria; firmicutes; clostridia; clostridiales; lachnospiraceae; blautia \\
\hline
\end{tabular}

RYGB = Roux-en-Y gastric bypass; SG = sleeve gastrectomy (vertical); SADS = single-anastomosis duodenal switch; OAGB = one-anastomosis gastric bypass; OTU = operational taxonomic unit; LDA = linear discriminant analysis..

Differently abundant OTUs across surgical groups. 


\section{Discussion}

Echoing the observation in humans, we found that rat models of bariatric surgical procedures led to significant and sustained weight loss and improvements in glucose metabolism. However, as summarized in Fig. 7, SG, SADS, RYGB, and OAGB incorporated different surgical approaches and resulted in differential effects on metabolic outcomes. Using this controlled animal model, we observed that OAGB met or exceeded the metabolic outcomes of RYGB, including increased weight loss, lipid reduction, and increased glucose-stimulated insulin and total GLP-1 release.

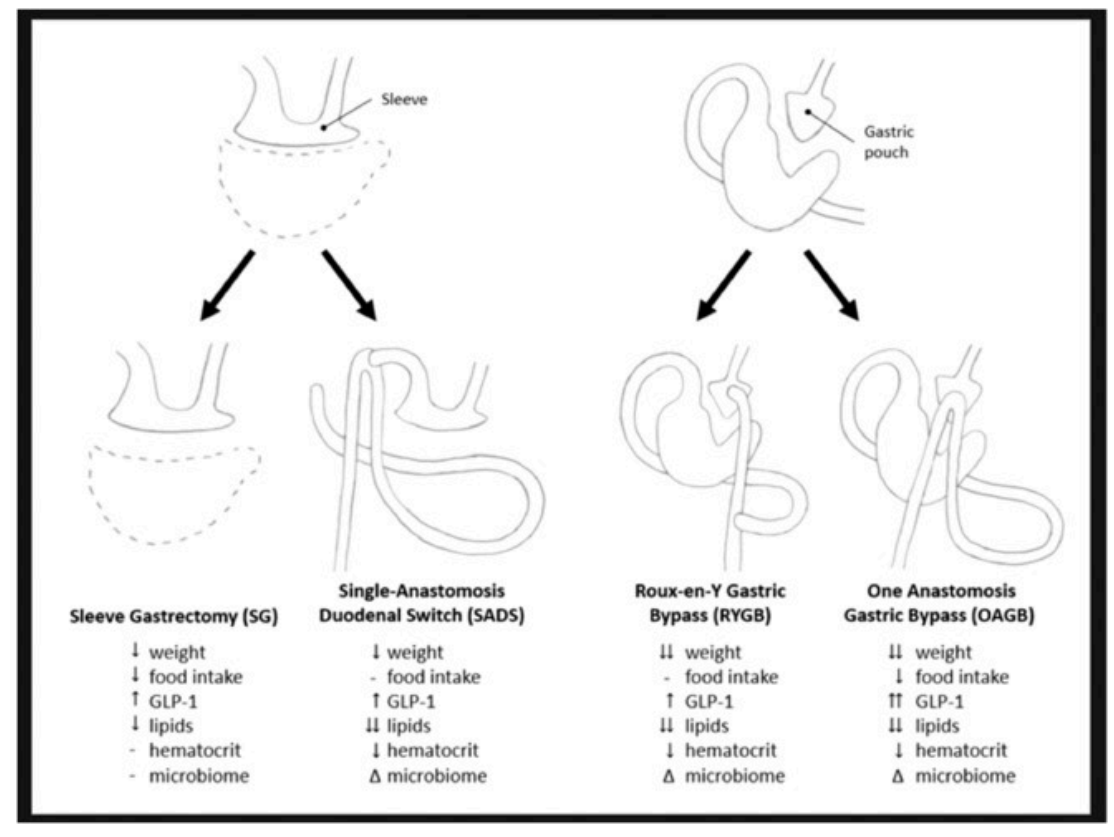

Fig. 7. Summary of surgical procedures and their major effects on metabolism.

We found that weight loss was significantly reduced in all surgical groups, with the most robust reductions in fat mass occurring in the OAGB and RYGB groups. Although a combined, clinical metaanalysis including all surgical procedures performed in this study is not available, separate clinical reports are available for comparison. In general, clinical and randomized controlled trials have indicated similar weight loss between RYGB and SG. $\underline{12}, \underline{13}$ However, some report greater fat mass loss in RYGB compared with $\mathrm{SG}, \underline{14}$ in agreement with our results. $A$ randomized control trial found that OAGB produced weight loss comparative to $S G, \underline{15}$ although $O A G B$ was associated with better glycemic control. $\underline{15}, 16$ A recent report on the weight outcomes of SADS versus RYGB indicated no differences in weight loss or diabetes remission. $\underline{17}$

OAGB and SG groups had decreased cumulative food intake compared with sham over the course of the experiment, contributing to weight loss. Although transient reductions in food intake were observed in all surgical groups, RYGB and SADS did not have any cumulative reduction in food intake, suggesting that these groups may experience an increase in energy expenditure or a decrease in nutrient absorption to account for the observed weight loss.

Notably, both OAGB and RYGB exhibit the most robust weight loss and leave the dissected stomach tissue in the body. Retaining the stomach tissue potentially allows gut hormones to be produced and pass readily into the circulation. OAGB also had the highest increases in GLP-1 levels in response to an 
oral glucose load. GLP-1 has robust actions on glucose regulation $\underline{18}$ and higher levels are associated with lowered glucose and insulin levels and improved metabolic health. ${ }^{19}$ Although improvement in fasting blood glucose was observed only in SG rats, glucose-induced insulin secretion was dramatically improved in all surgical groups.

An improved lipid profile was observed in all surgical groups, with the most profound decrease in plasma cholesterol and triglycerides observed in OAGB and SG, which could be attributed to the lower food intake also observed in these groups. Lower liver triglycerides reflect improved metabolism and lipid processing..$\underline{201}$ Although all surgical groups experienced a significant decrease in liver triglycerides, the most profound decreases in liver triglycerides occurred in surgical groups where nutrients were rerouted from the original path (e.g., RYGB, OAGB, and SADS). These data are consistent with the clinical data that, in general, indicate that RYGB reduces triglycerides more profoundly than $\mathrm{SG} . \underline{22}, \underline{23}$ Comparison studies examining triglyceride levels in OAGB and SADS are limited. One clinical study found that RYGB, OAGB, and SADS had similarly reduced triglyceride levels, consistent with the present data; however, there was no SG control to determine whether triglycerides were more robustly decreased in RYGB, OAGB, and SADS compared with SG. $\underline{17}$ However, 2 recent studies comparing OAGB to SG found no significant differences in triglycerides. $\underline{16}, \underline{23}$ When comparing preclinical rodent data with human clinical data, it is important to point out that clinical studies use plasma triglycerides, and in the present study, we found that plasma triglycerides were only significantly reduced compared with sham after OAGB and SG. Therefore, in the rat model, liver triglycerides may be a more appropriate indicator of clinical lipid metabolism postsurgery.

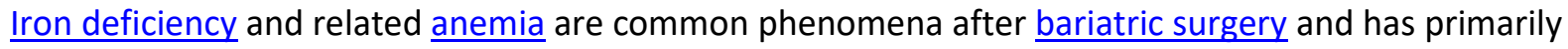
been attributed to bypassing the duodenum. ${ }^{24,} \underline{25}, \underline{26}, \underline{27}$ However, we find that except for RYGB, all surgical interventions reduced circulating iron levels. To maintain sufficient circulating iron levels, red blood cells are degraded by the macrophages to free hemoglobin-bound iron. Accordingly, hematocrit levels are decreased compared with sham in all bariatric surgical groups except for SG. An increased reticulocyte count is indicative of increased erythropoiesis at the level of the bone marrow and, combined with a reduced hematocrit level, is indicative of an increased erythrocyte turnover rate. Altogether, these data suggest that the low hematocrit levels observed in OAGB, SG, and SADS are caused by an increase of erythrocyte degradation to compensate for disrupted intestinal iron absorption. ${ }^{28}$ These rodent data are consistent with the clinical data in which iron deficiency and related anemia are common phenomena and more prevalent after duodenal bypass interventions than after SG. Although not directly assessed in the present study, these data are complementary to the nutritional malabsorption observed after clinical OAGB and SADS procedures. $\frac{5,29}{}$ Taken together, malabsorption is an important variable to consider when evaluating the metabolic outcome of a bariatric surgical procedure.

The fecal microbiome was significantly altered in OAGB, SADS, and RYGB surgical groups. These data point to the impact of rerouting nutrients and their effect on the microbiome independent of stomach tissue presence, incretins, the magnitude of weight loss, or changes in food intake. Indeed, a number of studies have shown a shift in the microbiota after RYGB. $\underline{10}, \underline{30}, \underline{31}$ Similarly, others have shown a significant

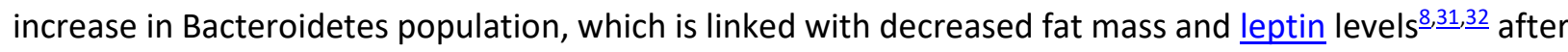
SG. However, in this data set, we did not observe a significant difference in the microbiome of rats after SG compared with sham. Part of this may be due to high weight variability in the SG rats as a regression analysis indicated that axis 2 of $\theta_{\text {Yc }}$ significantly predicted 15 -week postsurgical weight in the SG group. 
In the present study, we observe higher death rates after RYGB, OAGB, and SADS procedures compared with SG or sham, which is a potential limitation in our interpretations and conclusions. We suspect the loss to be due to the technical difficulty of performing the anastomosis technique in the relatively small rat model. Nevertheless, we have previously found that 6 to 7 animals per group is sufficient for robust metabolic analysis for animal studies of bariatric surgical procedures 33 and that the retention rate from the present study is in line with our previous observations.

In extrapolating our results to clinical practice, one key limitation of the present study is our surgical procedures for the OAGB and SADS do not exactly mirror those performed in humans. While we endeavored to closely recapitulate the clinical operations, we elected to standardize limb length across the RYGB, OAGB, and SADS produces, deviating from clinical practice. This standardization is important as it allows us to draw conclusions about how a specific manipulation to the gut, such as a single anastomosis (e.g., OAGB) and/or rerouting of nutrients in the context of a sleeve (e.g., SADS), affects metabolism without the added confounding variable of limb length. While limb length may be a contributing factor to metabolic outcomes, previous research has not found limb length to be a factor in weight loss obtained after RYGB. $\frac{34}{}$ Therefore, while these data cannot be directly applied to humans due to the methodic deviation from clinical OAGB and SADS, these data increase our understanding of how systemic changes to the gut can alter metabolism, which has marked clinical implications.

\title{
Conclusions
}

The results from these studies reveal that OAGB, SADS, RYGB, and SG surgical procedures have overlapping effects on a variety of parameters, including body fat, glucose regulation, insulin secretion, GLP-1 secretion, iron regulation, and lipid metabolism (Fig. 7). However, there remain important differences among these procedures that may contribute to different clinical effects. The ability to perform carefully controlled studies to directly compare the effects of multiple procedures is a major advantage of rodent models. The present data demonstrate that OAGB meets or exceeds the metabolic improvements induced by RYGB.

\section{Acknowledgments}

The authors thank the University of Michigan HMI core for processing and analyzing the fecal microbiome data. They also thank members of the Seeley laboratory for collecting plasma glucose levels during the glucose tolerance test.

\section{Disclosures}

RJS has received research support from Novo Nordisk, Janssen/Johnson \& Johnson, Zafgen, Medlmmune, and Kallyope and has served as a paid consultant to Ethicon Endo-Surgery/Johnson \& Johnson, Orexigen, Novo Nordisk, Daiichi Sankyo, Janssen/Johnson \& Johnson, Novartis, Paul Hastings Law Firm, Scohia and Kallyope Inc. DMA, SSE, NB, HFS, AM, AL, and MHT have no conflicts to declare.

\author{
Appendix. Supplementary materials \\ https://ars.els-cdn.com/content/image/1-s2.0-S155072891830532X-mmc1.docx \\ https://ars.els-cdn.com/content/image/1-s2.0-S155072891830532X-mmc2.docx
}




\section{References}

H Buchwald, DM Oien Metabolic/bariatric surgery worldwide 2011 Obes Surg, 23 (4) (2013), pp. 427436

R Peterli, B Wölnerhanssen, T Peters, et al. Improvement in glucose metabolism after bariatric surgery: comparison of laparoscopic Roux-en-Y gastric bypass and laparoscopic sleeve gastrectomy: a prospective randomized trial Ann Surg, 250 (2) (2009), pp. 234-241

B Laferrère, J Teixeira, J McGinty, et al. Effect of weight loss by gastric bypass surgery versus hypocaloric diet on glucose and incretin levels in patients with type 2 diabetes J Clin Endocrinol Metab, 93 (7) (2008), pp. 2479-2485

E Disse, A Pasquer, P Espalieu, et al. Greater weight loss with the omega loop bypass compared to the Roux-en-Y gastric bypass: a comparative study Obes Surg, 24 (6) (2014), pp. 841-846

A Sánchez-Pernaute, MA Herrera, ME Pérez-Aguirre, et al. Single anastomosis duodeno-ileal bypass with sleeve gastrectomy (SADI-S) One to three-year follow-up. Obes Surg, 20 (12) (2010), pp. 1720-1726

D. Sandoval Bariatric surgeries: beyond restriction and malabsorption Int J Obes, 35 (Suppl 3) (2011), pp. S45-S49

E Karra, A Yousseif, RL Batterham Mechanisms facilitating weight loss and resolution of type $\mathbf{2}$ diabetes following bariatric surgery Trends Endocrinol Metab, 21 (6) (2010), pp. 337-344

KK Ryan, V Tremaroli, C Clemmensen, et al. FXR is a molecular target for the effects of vertical sleeve gastrectomy Nature, 509 (7499) (2014), pp. 183-188

R Kohli, KD Setchell, M Kirby, et al. A surgical model in male obese rats uncovers protective effects of bile acids post-bariatric surgery Endocrinology, 154 (7) (2013), pp. 2341-2351

DM Arble, DA Sandoval, RJ Seeley Mechanisms underlying weight loss and metabolic improvements in rodent models of bariatric surgery Diabetologia, 58 (2) (2015), pp. 211-220

TE Sweeney, JM Morton The human gut microbiome: a review of the effect of obesity and surgically induced weight loss JAMA Surg, 148 (6) (2013), pp. 563-569

SH Chang, CR Stoll, J Song, et al. The effectiveness and risks of bariatric surgery: an updated systematic review and meta-analysis, 2003-2012 JAMA Surg, 149 (3) (2014), pp. 275-287

R Peterli, BK Wolnerhanssen, T Peters, et al. Effect of laparoscopic sleeve gastrectomy vs laparoscopic Roux-en-Y gastric bypass on weight loss in patients with morbid obesity: the SM-BOSS randomized clinical trial JAMA, 319 (3) (2018), pp. 255-265

B Woelnerhanssen, R Peterli, RE Steinert, et al. Effects of postbariatric surgery weight loss on adipokines and metabolic parameters: comparison of laparoscopic Roux-en-Y gastric bypass and laparoscopic sleeve gastrectomy-a prospective randomized trial Surg Obes Relat Dis, 7 (5) (2011), pp. 561-568

S Seetharamaiah, O Tantia, G Goyal, et al. LSG vs OAGB-1 year follow-up data-a randomized control trial Obes Surg, 27 (4) (2017), pp. 948-954

WJ Lee, K Chong, YH Lin, JH Wei, SC Chen Laparoscopic sleeve gastrectomy versus single anastomosis (mini-) gastric bypass for the treatment of type 2 diabetes mellitus: 5-year results of a randomized trial and study of incretin effect Obes Surg, 24 (9) (2014), pp. 1552-1562

WJ Lee, KT Lee, K Kasama, et al. Laparoscopic single-anastomosis duodenal-jejunal bypass with sleeve gastrectomy (SADJB-SG): short-term result and comparison with gastric bypass Obes Surg, 24

(1) (2014), pp. 109-113 
P Nadkarni, OG Chepurny, GG Holz Regulation of glucose homeostasis by GLP-1 Prog Mol Biol Transl Sci, 121 (2014), pp. 23-65

F Rubino, A Forgione, DE Cummings, et al. The mechanism of diabetes control after gastrointestinal bypass surgery reveals a role of the proximal small intestine in the pathophysiology of type 2 diabetes Ann Surg, 244 (5) (2006), pp. 741-749

AM Coulston, CB Hollenbeck, AL Swislocki, GM Reaven Persistence of hypertriglyceridemic effect of low-fat high-carbohydrate diets in NIDDM patients Diabetes Care, 12 (12) (1989), pp. 94-101

PD Berk, EC Verna Nonalcoholic fatty liver disease: lipids and insulin resistance Clin Liver Dis, 20 (2) (2016), pp. 245-262

SP Heffron, A Parikh, A Volodarskiy, et al. Changes in lipid profile of obese patients following contemporary bariatric surgery: a meta-analysis Am J Med, 129 (9) (2016), pp. 952-959

$\mathrm{N}$ Quezada, F Leon, A Jones, et al. High frequency of internal hernias after Roux-en-Y gastric bypass Obes Surg, 25 (4) (2015), pp. 615-621

A Alexandrou, E Armeni, E Kouskouni, et al. Cross-sectional long-term micronutrient deficiencies after sleeve gastrectomy versus Roux-en-Y gastric bypass: a pilot study Surg Obes Relat Dis, 10 (2) (2014), pp. 262-268

MC Chen, YC Lee, WJ Lee, HL Liu, KH Ser Diet behavior and low hemoglobin level after laparoscopic mini-gastric bypass surgery Hepatogastroenterology, 59 (120) (2012), pp. 2530-2532

Y Kwon, HJ Kim, E Lo Menzo, et al. Anemia, iron and vitamin B12 deficiencies after sleeve gastrectomy compared to Roux-en-Y gastric bypass: a meta-analysis Surg Obes Relat Dis, 10 (4) (2014), pp. 589-597

RF Kushner Micronutrient deficiencies and bariatric surgery Curr Opin Endocrinol Diabetes Obes, 13 (5) (2006), pp. 405-411

JM Higgins Red blood cell population dynamics Clin Lab Med, 35 (1) (2015), pp. 43-57

M Carbajo, M Garcia-Caballero, M Toledano, et al. One-anastomosis gastric bypass by laparoscopy: results of the first 209 patients Obes Surg, 15 (3) (2005), pp. 398-404

AP Liou, M Paziuk, JM Luevano Jr., et al. Conserved shifts in the gut microbiota due to gastric bypass reduce host weight and adiposity Sci Transl Med, 5 (2013), p. 178ra41

R Murphy, P Tsai, M Jullig, et al. Differential changes in gut microbiota after gastric bypass and sleeve gastrectomy bariatric surgery vary according to diabetes remission Obes Surg, 27 (4) (2017), pp. 917-925

JP Furet, LC Kong, J Tap, et al. Differential adaptation of human gut microbiota to bariatric surgeryinduced weight loss: links with metabolic and low-grade inflammation markers Diabetes, 59 (12) (2010), pp. 3049-3057

AP Chambers, L Jessen, KK Ryan, et al. Weight-independent changes in blood glucose homeostasis after gastric bypass or vertical sleeve gastrectomy in rats Gastroenterology, 141 (3) (2011), pp. 950958

NV Christou, D Look, LD Maclean Weight gain after short- and long-limb gastric bypass in patients followed for longer than 10 years Ann Surg, 244 (5) (2006), pp. 734-740 